\title{
Pharmacogenetics and Personalized Medicine in Hematology
}

\author{
Hara Prasad Pati $^{1} \cdot$ Prashant Sharma $^{2}$
}

Received: 28 June 2017/ Accepted: 28 June 2017/Published online: 4 July 2017

(C) Indian Society of Haematology \& Transfusion Medicine 2017

Genetic variation among human beings has a significant impact on disease susceptibility as well as treatment outcomes. It is now recognized that the human genome, despite its $99.5 \%$ sequence homology within the species, is unique for every individual because of the thousands of single nucleotide polymorphisms, insertion-deletion mutations and copy number variations as well as alternative splicing patterns and variable gene expression profiles that individuals display [1]. This genetic heterogeneity is often imperceptible at the phenotypic level in healthy persons, but research has revealed its associations with several diseases like diabetes mellitus and many cardiac, neurological and psychiatric illnesses [2]. It also has major implications in hematological disorders, and precision and personalized medicine is considered one of the frontier areas of translational research today. Salient examples include the incorporation of CYP2C9 and VKORCl genotypes in oral anticoagulant dosing algorithms and the influences of thiopurine S-methyltransferase (TPMT) and methylene tetrahydrofolate reductase (MTHFR) genotypes on the safety and efficacy of 6-mercaptopurine and methotrexate therapies respectively in acute lymphoblastic leukemia [3].

Tumour genomes are even more heterogeneous and they are the subject of multiple articles in this issue. Tripathi et al [4] report the patterns of $A B L$ tyrosine kinase domain mutations in 40 chronic myelogenous leukemia (CML)

\footnotetext{
Hara Prasad Pati

harappati@yahoo.co.in

Prashant Sharma

prashant.sh@gmail.com

New Delhi, India

2 Chandigarh, India
}

patients with loss/non-attainment of response to imatinib. Sixteen of the 40 had detectable mutations, with M351T, Y253H and H396R being the commonest. The remaining cases might have harboured $\mathrm{SH} 2 / \mathrm{SH} 3$ auto-inhibitory domain mutations, possessed amplified $\mathrm{Ph}$-chromosomes or could have displayed $B C R / A B L 1$-independent mechanisms like inhibited apoptotic signalling, increased drug efflux via membrane pumps or alterations in transcription factors [5]. In any event, identification of the genotypic differences in these patients vis-à-vis imatinib-responsive CML cases provided evidence-based grounds to switch to newer-generation TKIs.

Regions of the genome that do not encode protein sequences are not inconsequential in determining health and disease. This is brought out elegantly by two papers in this issue. In the first, Dehkordi et al [6] blocked the microRNA mir-222 in a chronic lymphocytic leukemia (CLL) cell line leading to reduced lymphocytic survival. For this, they used locked nucleic acids (LNAs), a proprietary technology that enhance hybridization by increasing the melting temperature of oligonucleotides. LNAs are frequently employed by molecular biology firms to manufacture sensitive and specific DNA microarrays and FISH and qPCR probes.

In another paper on non-coding RNAs, Emamdoost et al [7] studied miR-125a-3p-mediated inhibition of TIM-3, an immune checkpoint receptor that is a promising candidate for targeted therapies in cancer. These authors used a human promyelocytic leukemia cell line (HL-60) to demonstrate the strong silencing of TIM- 3 expression by both qPCR and flow cytometry (i.e. at the mRNA and protein levels). This paper is important since TIM-3 ameliorates Th1 and $\mathrm{Tc} 1 \mathrm{~T}$ cell responses and induces tumour antigen-specific tolerance [8]. Pre-therapy TIM-3 levels in AML patients are heterogeneous and overlap extensively 
with normal controls [9] and whether determination of expression intensity will be required remains to be studied.

Our understanding of molecular heterogeneity at the genome level in AML, in general, far exceeds that of other hematological neoplasms. A landmark NEJM publication in 2016 [10] sequenced 111 genes in over 1500 AML patients and revealed a staggering 5234 driver mutations in 76 genes or regions. The proposed "genomic" classification of AML identified new molecular subgroups including AML with mutations in genes encoding chromatin and/or RNA-splicing factors; AML with TP53 mutations and/or chromosomal aneuploidies and AML with IDH2R172 mutations. The race to design, test and market therapies directed against these "targetable" molecular lesions is already on.

But finally, we must remember that genetics may not explain everything, as brought out by a Mexican study in this issue. Vallejo-Villalobos et al [11] found that activated protein $\mathrm{C}$ resistance (APCR) that remained unexplained in patients with thrombosis even after testing for factor $\mathrm{V}$ Leiden, increased factor VIII levels and anti-phospholipid antibodies, actually correlated with the intake of directacting oral anticoagulants in the last $12-24 \mathrm{~h}$. Factitious over-estimation of APCR is a known interference of both direct thrombin and Xa inhibitors [12]. The importance of integrating clinical information in laboratory assay interpretation therefore cannot be overemphasized, even in this era of high-end genomics.

\section{References}

1. Levy S, Sutton G, Ng PC, Feuk L, Halpern AL, Walenz BP et al (2007) The diploid genome sequence of an individual human. PLoS Biol 5:e254
2. Perkel J (2015) Making sense of our variation. Biotechniques 59:262-267

3. Davies SM (2006) Pharmacogenetics, pharmacogenomics and personalized medicine: are we there yet? Hematol Am Soc Hematol Educ Program 1:111-117

4. Tripathi AK, Verma SP, Kumar N (2016) Mutation analysis in chronic myeloid leukemia patients in chronic phase on imatinib having delayed achievement of milestones or loss of response. Indian J Hematol Blood Transfus. doi:10.1007/s12288-016-0755-y

5. Huang R, Kang Q, Liu H, Li Y (2016) New Insights into the molecular resistance mechanisms of chronic myeloid leukemia. Curr Cancer Drug Targets 16:323-345

6. Dehkordi KA, Chaleshtori MH, Sharifi M et al (2016) Inhibition of MicroRNA miR-222 with LNA inhibitor can reduce cell proliferation in B chronic lymphoblastic leukemia. Indian $\mathrm{J}$ Hematol Blood Transfus. doi:10.1007/s12288-016-0694-7

7. Emamdoost F, Khanahmad H, Ganjalikhani-hakemi $M$ et al (2016) The miR-125a-3p inhibits TIM-3 expression in AML cell line HL-60 in vitro. Indian J Hematol Blood Transfus. doi:10. 1007/s12288-016-0733-4

8. Anderson AC (2014) Tim-3: an emerging target in the cancer immunotherapy landscape. Cancer Immunol Res 2:393-398

9. Roth CG, Garner K, Eyck ST, Boyiadzis M, Kane LP, Craig FE (2013) TIM3 expression by leukemic and non-leukemic myeloblasts. Cytom B Clin Cytom 84:167-172

10. Papaemmanuil E, Gerstung M, Bullinger L et al (2016) Genomic classification and prognosis in acute myeloid leukemia. N Engl $\mathrm{J}$ Med 374:2209-2221

11. Vallejo-Villalobos MF, León-Peña A, León-González M et al (2016) Primary thrombophilia in México XI: activated protein C resistance phenotypes are multifactorial. Indian J Hematol Blood Transfus. doi:10.1007/s12288-016-0767-7

12. Adcock DM, Gosselin R (2015) Direct oral anticoagulants (DOACs) in the laboratory: 2015 review. Thromb Res 136:7-12 